\title{
Persistence of Simple Substances
}

\author{
Markku Keinänen \\ Post-doctoral Researcher, Academy of Finland and University of Turku \\ Department of Behavioural Sciences and Philosophy \\ 20014 University of Turku \\ Finland \\ Jani Hakkarainen \\ Post-doctoral Researcher, Academy of Finland and University of Tampere \\ Department of History and Philosophy/Philosophy \\ 33014 University of Tampere
}

Finland

\begin{abstract}
In this paper, we argue for a novel three dimensionalist (3D'ist) solution to the problem of persistence, i.e., cross-temporal identity. We restrict the discussion of persistence to simple substances, which do not have other substances as their parts. The account of simple substances employed in the paper is a trope-nominalist Strong Nuclear Theory (SNT), which develops Peter Simons' trope nominalism. Regarding the distinction between three dimensionalism (3D) and four dimensionalism (4D), we follow Michael Della Rocca's formulation, in which 3D explains persistence in virtue of same entities and 4D in virtue of distinct entities (temporal parts). SNT is a 3D'ist position because it accounts for the persistence of simple substances in virtue of diachronically identical 'nuclear' tropes. The nuclear tropes of a simple substance are necessary for it and mutually rigidly dependent but distinct. SNT explains qualitative change by tropes that are contingent to a simple substance. We show that it avoids the standard problems of 3D: temporal relativization of ontic predication, Bradley's regress, and coincidence, fission and fusion cases. The temporal relativization is avoided because of the analysis of temporary parts that SNT gives in terms of temporal sub-location, which is atemporal part-whole relation.
\end{abstract}

\section{Introduction}




\subsection{Problem}

Persistence has been one of the liveliest discussions in new analytic metaphysics. Persistence may be understood in a broad or narrow sense. In the broad sense, it means identity over time in general, i.e., $a_{1}$ at $t_{1}$ and $a_{2}$ at $t_{2}$ are numerically identical entities. The narrow sense qualifies cross-temporal identity with qualitative change: $a_{1}$ and $a_{2}$ are still identical although there is a difference in their (intrinsic) properties.

The problem of persistence or what is essential to it have been put differently by different philosophers. In this paper, we take that the problem of persistence concerns primarily metaphysical explanation: what accounts for or grounds the persistence of an entity. This concerns both the broad and the narrow sense of persistence.

Regarding the narrow sense, diachronic identity with qualitative change, we follow Roxanne Marie Kurtz's approach rather closely. According to Kurtz (2006, 12-13, cf. also 2,3), the problem of persistence consists in the negotiation between the following three theses:

1. 'alteration':

"Any object that changes is the proper subject of the incompatible properties involved in the change."

\section{2. 'survival':}

"If an object persists through change, then the object existing before the change is numerically identical to the one existing after the change."

3. 'atemporal instantiation'/temporary intrinsics:

"If an object is the proper subject of a property, then (i) the object has that property, and (ii) facts about time and tense are irrelevant to the truth of the proposition that the object has that property."

According to Kurtz, these three theses entail a contradiction if they are conjoined with three non-negotiable theses about persistence and change. These three non-negotiable theses are fairly incontestable: (4) objects cannot possess incompatible properties, (5) change involves incompatible properties, and (6) objects persist through change. Together (1), (2), (3), (5) and (6) seem to entail that an object surviving change has incompatible properties. But this is contradictory to (4): nothing can have incompatible properties. So in Kurtz's view, the problem of persistence in the narrow sense is the negotiation of theses (1)-(3) because (4)-(6) are non- 
negotiable. For instance, the contradiction may be avoided by relativizing incompatible properties to time, which negotiates the third thesis. (Kurtz 2006, 2, 14-15)

In principle, there can be many kinds of entities that persist: substances, processes and persons, for instance. For the sake of simplicity, in this paper we restrict our discussion to concrete particulars and especially to simple individual substances. Simple substances do not have other individual substances as their proper parts. Basically, they are fundamental physical particles such as quarks and electrons. So this paper does not discuss complex 'medium-sized dry goods': chairs, tables, etc. Explaining their persistence is a matter of future research.

Metaphysical positions on persistence are typically divided into two main categories: three dimensionalism (3D) and four dimensionalism (4D). There are various ways to make this distinction in the literature. It is typically characterised as follows: 4D explains persistence by temporal parts, which 3D does not accept but states that substances persist by being 'wholly present' during their time of existence. However, we find this problematic because even Katherine Hawley's (2001, 26-28) description of 'whole presence', which is the acutest perhaps in the literature, has problematic involvements.

Hawley contrasts whole presence at distinct moments (as a multiple temporal location at these moments as a whole) with being temporally extended in the time interval comprising these moments (as a single temporal location at the interval), i.e., begin spread over the interval. While the basic notion of parthood for the temporally extended objects (perdurants) is atemporal, the appropriate notion of parthood for the wholly present objects (endurants) is a temporally relativized one. Thus, according to Hawley, the appropriate notion of parthood for endurants is necessarily relativized to time. Endurant $e$ is wholly present at each moment of its existence because $e$ has at time $t$ only the parts that $e$ has at that time.

The problematic involvements in Hawley's way of putting the distinction are twofold. First, some endurants can be considered as temporally extended simples. Therefore a 3D'ist does not have to consider them multiply temporally located entities as Hawley claims. ${ }^{1}$ Secondly, this paper shows that there is a coherent 3D that does not have to relativize parthood to time.

Since describing 3D in terms of 'whole presence' is not satisfactory, we prefer Michael

$1 \quad$ Another interesting recent attempt to explicate whole presence is by Crisp \& Smith (2005). However, because their definition entails that extended simples are wholly present at each subregion of the region of spacetime they occupy $(2005,342 \mathrm{ff}$.), it fails to deal with spatio-temporally extended simples in a satisfactory fashion. 
Della Rocca's $(2009)^{2}$ characterisation of the distinction between 3D and 4D, which does not suffer from the same problems. It is meant to be as comprehensive and general as possible: to encompass every internally coherent position on persistence notwithstanding their other possible dismerits such as redundancy. It is also neutral on the kind of persisting entities. According to Della Rocca, 4D states that entities persist in virtue of distinct entities, whereas in 3D, entities persist in virtue of same entities (objects persist because of identical entities). Della Rocca understands the problem of persistence in the above-described manner. Persistence is cross-temporal identity with or without qualitative change. The problem of persistence is to explain this cross-temporal identity of some entity or kind of entities. Thus, 'in virtue of' in the distinction refers to metaphysical explanation: to the entity or entities that ground persistence. The identical entities in 3D might be, for example, the objects themselves or their proper parts. The moot point is that (at some point) we must arrive at entities whose persistence is ungrounded: for instance, the proper parts of objects explaining their persistence and which persist by remaining identical through the time of their existence, i.e., without dividing into temporal parts. ${ }^{3}$

Basically, the distinct entities explaining persistence in 4D are temporal parts. So Lewis' (1986) worm theory is a form of 4D, which is also called Perdurantism (Kurtz 2006, 5-6). Persisting objects perdure: they are mereological sums of temporal parts. Another form of 4D may be called Exdurantism (Kurtz 2006, 7). According to Hawley's (2001) and Theodore Sider's (2001) (different) exdurantist 4D's, objects are identified with temporal parts or stages. That is why it is normally called 'stage theory'. In Sider's stage theory, it is temporal counterpart relations between stages that determine the stages with which a substance may be identified and that explain persistence.

3D or Endurantism is a wide category: it covers many different theories. For example, a

2 Della Rocca, M. (2009). Primitive Persistence and the Impasse between Three-dimensionalism and Fourdimensionalism. Manuscript.

3 Although Jonathan Lowe (1998, 98ff.) characterizes the persistence of objects in terms of whole presence, he also introduces 'substantial constituents approach' in order to explain metaphysically the persistence of continuant substances (Lowe 1998, ch.5), which is independent of the characterisation in terms of whole presence. According to it, substances persist by virtue of the persistence of their constituent substances but substances must be eventually constituted by primitive substances which persist by just remaining identical through the time of their existence. 
substance may be said to persist because it remains identical without having temporal parts or a substance is cross-temporally identical at $t_{1}$ and $t_{2}$ because its substratum remains identical.

The difference between 3D and 4D is often formulated in terms of the acceptance of temporal parts (e.g. Simons 1987, 121ff.): 4D'ists assume that all non-instantaneous entities, concrete individual objects included, have proper temporal parts, while 3D'ists deny that concrete objects (or substances) have any. Yet 3D'ists and 4D'ists alike can accept such concrete entities as processes, which consist of distinct temporal parts if temporally extended.

However, this characterisation is not satisfactory for two reasons. First, there can be temporally extended simple processes, which do not have any further temporal parts. Both 3D'ists and 4D'ists can accept such entities. The second reason may be put by restricting to substances. According to Della Rocca's distinction, it is possible for a 3D'ist to admit that substances have proper temporal parts - just as long as they do not persist in virtue of them. ${ }^{4}$

Hence, rather than being committed to an outright rejection of the temporal parts of entities, following Della Rocca, we suggest that the defining character of 3D is that it does not account for persistence by means of temporal parts. Yet 3D as such is neutral on the existence of proper temporal parts. According to it, substances, for example, persist because of entities that do not have temporal parts (while substances may have proper temporal parts). By contrast, processes and perdurant objects advocated by 4D'ists persist due to having minimal temporal parts at the distinct moments of their existence. ${ }^{5}$

According to some endurantists, a central part of the problem of persistence is about the persistence criteria or conditions of objects. Do they have determinate persistence conditions? How are these persistence conditions determined? (e.g., Lowe 1998, 107-109) In Lowe's framework, diachronic identity criteria are metaphysical principles that account for the persistence of complex objects by means of their proper parts arranged in a certain way. By contrast, primitive substances do not have any informative identity criteria. Our theory delivers

$4 \quad$ Cf. Kurtz (2006, 10). Sider's (2001, sec. 3.3) example of a temporal part of an endurant, i.e., a lump of clay, is a statue, which is made of exactly the same micro-particles as the lump but exists only an instant moment of lump's existence. Here, we do not take any stance on Siders' example: whether it is based on acceptable mereological assumptions or not, for instance.

$5 \quad$ Standard 4D'ists such as Sider (2001) and Hawley (2001) assume that the minimal temporal parts of objects are instantaneous and do not any have temporal extension. Hawley $(2001,28)$ admits, though, that there can be 'hypothetical 4D'ists' claiming that the minimal temporal parts are temporally extended simples. Sider agrees $(2001,60)$. 
certain persistence conditions for simple substances but we do not take up the general problem about identity conditions in this paper. Our focus is on the metaphysical explanation of crosstemporal identity with or without qualitative change.

\subsection{Aim}

The aim of this paper is to argue for a novel version of 3D. We find that the structure of individual substances has not received sufficient attention on the 3D'ist side of the (contemporary) literature, which has given unnecessary advantage to 4D in this respect. We defend a 3D that gains its insight from a developed theory of simple individual substances. That theory is one of the authors', Author A's trope-bundle theory, which we call Strong Nuclear Theory (SNT). It elaborates on Peter Simons's (1994) trope-nominalist account. The basic idea in the SNT is that simple individual substances consist of mutually dependent tropes, which are particular properties. It is a 3D'ist account of persistence because simple substances persist in virtue of cross-temporally identical tropes, which do not have temporal parts.

We are not going to argue against 4D. In this paper, we merely defend a 3D (SNT) for the standard objections to 3D: (1) temporal relativization of temporary intrinsics and mereological relations, (2) Bradley's regress, (3) coincidence, fission and fusion cases. Our focus is on the first two as the third concerns mainly complex individual substances and persons, which are not the subject of this paper.

Temporal relativization of temporary intrinsics is a genuine problem for three reasons (Kurtz 2006, 20-21). First, it seems that there could hardly be qualitative change/alteration without incompatible temporary intrinsics. Secondly, intuitively, temporary properties would not count as intrinsic if they were relativized to time because entities have intrinsic properties merely due to themselves without mediation through something extrinsic to them. Thirdly, temporal relativization of temporary intrinsics is closely related to Bradley's regress. If temporary intrinsics are relativized to time, the analysis of ontic predication (the relation between substances and their properties) involves relations to times and Bradley's regress, which concerns relations, may threaten its analysis. Bradley's regress is the problem that if ontic predication is analyzed by external relations that are further entities to their relata, it creates a vicious infinite regress. 
We will show that the SNT does not suffer from these problems. (1) It provides atemporal analysis of ontic predication because the formal ontological dependencies that it employs are atemporal and it is not tropes but certain kinds of trope aggregates (bundles) that are basic entities figuring in spatio-temporal relations. As will be explained below, because trope aggregates are mereologically constant, the part-whole relations between individual tropes and these trope aggregates are not temporally relativized. (2) Relations involved in the SNT are either relational tropes or formal relations. Formal relations required in the construction of substances are existential-dependence relations and mereological relations. Formal relations are not further entities to their relata. Relational tropes grounding the spatiotemporal location of substances are bound to their bearers by the formal relation of rigid dependence. Thus, Bradley's regress is halted by formal relations, which are not further entities to their relata.

Current discussion on persistence typically assumes close parallels between the logical structure of truth-bearers and the categorial (or ontic) structure of truth-makers. We avoid assuming such parallels by focusing on ontic predication and truth-makers. Propositions attributing features to objects can be temporally modified, while the entities making propositions true (e.g. tropes) need not be time relativized.

We show therefore that 3D can accept Kurtz's thesis 3 when it is properly modified or specified. Focusing on ontic predication, it should say simply that having properties is not relativized to time. Let this be thesis 3.1 and Kurtz's original proposition 3.2. Our account solves the problem of persistence in the narrow sense according to her description by means of specifying this third thesis together with the fourth that objects cannot have incompatible properties. As will be seen below, it is possible for simple substances to have properties that would exclude each other at the same time - be incompatible - because simple substances are mereologically inconstant. Yet we do not have to reject 3.1: they can do that without relativizing ontic predication to time.

According to the standard 4D'ist theories, an object has a spatio-temporal part corresponding to each point of space-time it occupies ('geometric correspondence principle'). ${ }^{6}$ By contrast, the 3D'ist theory advocated here permits that objects are spatio-temporally

6 Cf. Hawthorne (2008, 264, 268-269). The term 'geometric correspondence principle' is coined by Simons (2004). Hawley (2001, 48-50) argues that "stages are as fine-grained as possible change", a stage corresponding to each moment of the existence of a four-dimensional perdurant. 
extended simples, i.e., spatio-temporally extended objects that do not have any further objects as their proper parts. Simple physical microparticles form natural examples of spatiotemporally extended simples.

We will not discuss metaphysics of time in this article, but we are contented to assume Eternalism and tenseless theory of time. ${ }^{7}$

The paper has a six-part structure. After the Introduction, we outline the SNT in the second section. Section 3 forms the discussion of the problem of the temporal relativization of ontic predication and in the fourth section we show that the SNT avoids Bradley's regress. In Section 5, we consider coincidence, fission and fusion cases briefly. The paper is concluded by summarising it.

\section{Overview of Strong Nuclear Theory}

In the traditional substance/property or substance/accident ontology, it is substances that are fundamental and ontologically independent entities and properties depend on or inhere in them. Substances are also meant to survive alteration or qualitative change. They are proper subjects of change, they persist through it. Although in the SNT substances are still proper subjects of qualitative change, what remains the same in alteration, the SNT breaks with this tradition by building substances out of particular properties, i.e., tropes. According to the SNT, tropes rather than substances - are fundamental building blocks of the universe.

In this section, we first describe how simple substances are construed in the SNT. Then we discuss the issue of spatio-temporal location of substances and their parts. This leads us to the question of temporary intrinsics in the next section.

Strong Nuclear Theory is a trope-bundle theory of simple substances, which are analyzed as the bundles of property tropes. According to all trope-bundle theories, tropes are particular properties. This involves that tropes are concrete, i.e., spatio-temporal particulars. As basic particulars, tropes have determinate identity and counting conditions. Moreover, tropes are categorially simple: each trope is either simple or all parts of tropes are further tropes. Tropes are also thin in nature: each trope determines a single feature of the object possessing

$7 \quad$ The consistency of Eternalism with 3D is defended by Mellor (1981), Haslanger (1989) and Van Inwagen (1990). 
that trope. Finally tropes can occur as spatio-temporally co-located (compresent) with each other. $^{8}$

Rival trope-bundle theories of substance offer us different ways to construct substances in terms of trope bundles. While according to independence theories (Williams 1953a, b; Campbell 1990) substances are bundles of mutually compresent tropes, dependence theories (Simons 1994; Denkel 1996, 1997) use the formal relations of rigid and generic dependence in constructing substances. ${ }^{9}$ Strong Nuclear Theory is a dependence theory: in it, there are two types of tropes, necessary and contingent. According to the SNT, what is called 'the nucleus' of substance $i$ is formed by one or more necessary tropes. If the substance has two or more of these 'nuclear tropes', they are strongly rigidly dependent (SRD) on each other. Each simple substance has one or more nuclear tropes. Nuclear tropes are necessary to substance $i$ and determine the kind $\mathrm{K}$ to which $i$ belongs. In order to guarantee the diversity of the distinct tropes constituting the nucleus, the SNT assumes that each nuclear trope falls under a distinct highest determinable. ${ }^{10}$ A good prima facie example of a simple substance is a down quark or an electron. The nuclear tropes such as a rest mass trope of $9.10938215 \times 10^{-31} \mathrm{~kg}$ and a charge trope of $-e$ determine the necessary features of the electron, which are determinate physical quantities.

It is not necessary in the SNT that a simple substance has any contingent tropes as its parts. It might be that nuclear tropes are sufficient. For it is possible that they already occupy the tropes of the certain distinct determinable kinds $\mathrm{D}_{1}, \ldots \mathrm{D}_{\mathrm{k}}$, on which the nuclear tropes of substance of kind $\mathrm{K}$ are generically dependent and which the substance of kind $\mathrm{K}$ must have. If this is not the case, it has contingent tropes occupying the rest of $D_{1}, \ldots D_{k}$. These contingent tropes are strongly rigidly dependent on the nuclear tropes. However, this dependence is merely one-sided; the nuclear tropes are strongly generically dependent (SGD) on the kinds of contingent tropes. It also follows that all the tropes of a simple substance are strongly

\footnotetext{
8 See Simons (2000, sec.6); Maurin (2002, ch.2) and Author () for further discussion.

9 Let ' $\leq$ ' be improper parthood relation between distinct entities. Trope $u$ is strongly rigidly dependent on trope $s$ if $\operatorname{SRD}(\mathrm{u}, \mathrm{s}) \equiv \neg(\square \mathrm{E} ! \mathrm{s}) \wedge \square((\mathrm{E} ! \mathrm{u} \rightarrow \mathrm{E} ! \mathrm{s}) \wedge \neg(\mathrm{s} \leq \mathrm{u}))$ holds. Trope $x$ of kind $\mathrm{P}$ is strongly generically dependent on trope $y$ of kind $\mathrm{R}$ if $\operatorname{SGD}(\mathrm{P}(\mathrm{x}), \mathrm{R}(\mathrm{y})) \equiv \square \forall \mathrm{x} \square(\mathrm{Px} \rightarrow \square(\mathrm{E} ! \mathrm{x} \rightarrow \exists \mathrm{y}(\mathrm{Ry} \wedge \neg(\mathrm{y} \leq \mathrm{x})))) \wedge \diamond \exists \mathrm{x} \operatorname{Px} \wedge$ $\neg \square \forall \mathrm{x}$ Rx holds. See Simons (1987, $294 \mathrm{ff}$.) for the more detailed discussion of these dependence relations.

10 In contradistinction to the SNT, Simons's (1994) Nuclear Theory does not constrain what different kinds of tropes can form a nucleus of a substance. A nucleus of substance can be constituted solely, e.g., by mass tropes, or in any case, by several tropes that belong to the same determinable kind.
} 
generically dependent on the tropes of each other's kinds because the substance of kind K must have the tropes of the certain distinct determinable kinds $\mathrm{D}_{1}, \ldots \mathrm{D}_{\mathrm{k}}{ }^{11}$ The tropes strongly rigidly dependent on the nuclear tropes of the simple substance constitute it and it contains no other tropes. In other words, these tropes are the proper parts of the substance. In general, according to the SNT, tropes are the proper parts of a simple substance if and only if they are strongly rigidly dependent only on the nuclear tropes of the substance. ${ }^{12}$

Hence, according to the SNT, each substance $i$ is an individual formed by the tropes rigidly dependent on its nuclear tropes. That the bundle of such tropes forms an individual is an additional assumption made by the SNT. Further individuals the trope-bundles form are $c$ bundles and $n$-bundles (cf. below).

It does not follow from the strong rigid and generic dependence between tropes that they are spatio-temporally co-located because as formal relations these dependencies are neutral on spatio-temporal location. For persistence, it is necessary, however, that the tropes of a substance cannot be dispersed over space-time; otherwise contingent tropes could be temporally distinct from the simple substance, for instance. Next we will show that this can happen for neither nuclear nor contingent tropes. We can keep the simple substances together as trope bundles.

The argument for the compresence (spatio-temporal co-location) of the nuclear tropes of a substance with each other and their bundle is not complicated from the SNT. The bundle of the nuclear tropes of each substance $i$, which we call $n$-bundle of $i$, is bound together by strong rigid dependence. One of the important points in the SNT is that it is this trope aggregate that is figuring in the basic spatio-temporal relations. The $n$-bundle is a minimal entity instantiating the basic spatio-temporal relations: since its constituent nuclear tropes do not instantiate the basic spatio-temporal relations, they do not have independent location. Therefore the spatiotemporal location of the $n$-bundle determines the location of each nuclear trope. Since the $n$ bundle must have some spatio-temporal location as a concrete entity, this location is also the location of every nuclear trope in the $n$-bundle. They have the same spatio-temporal location; if

11 A trope belongs necessarily to the determinable kind D to which it belongs. Therefore, by being strongly rigidly dependent on certain other tropes, a nuclear trope is also strongly generically dependent on the tropes that belong to determinable kinds of the tropes on which it is rigidly dependent.

12 Both strong rigid dependence and proper parthood are primitive formal relations, but the latter is needed in the characterization of the former. 
it were possible that the nuclear tropes had different location than the $n$-bundle, the $n$-bundle could not determine their location. Further, the existence of the nuclear tropes entails that they are proper parts of the $n$-bundle. Thus, if nuclear tropes exist, they are necessarily compresent with each other and the $n$-bundle. The substance cannot change its nuclear tropes during the time of its existence either because they are necessary for it. Hence, substance $i$, the $n$-bundle and the nuclear tropes exist only when each of them exists. Spatially, the $n$-bundle and the nuclear tropes have exactly the same location. ${ }^{13}$

Regarding persistence, this means that it is the nuclear tropes of a simple substance that explain or ground its cross-temporal identity. Since this happens in virtue of the persistence of the nuclear tropes, i.e., in virtue of the same entities, the SNT is a 3D'ist theory of persistence.

Many substances possess contingent tropes, which are one-sidedly (strongly) rigidly dependent on nuclear tropes. According to the SNT, the trope aggregates formed by each single contingent trope $u$ of substance $i$ and the nuclear tropes of $i$, which we call $c$-bundles, figure in the basic spatio-temporal relations as well as $n$-bundles. ${ }^{14}$ The location of the $c$-bundle determines the location of the contingent trope $u$. Therefore the relation between the $c$-bundle and the $n$-bundle is relevant for the relation between trope $u$ and the $n$-bundle. So we have to consider two principles regulating the relation between the $c$-bundle and the $n$-bundle. They determine the temporal and spatial location of the $c$-bundle relative to the location of the $n$ bundle respectively.

As a trope aggregate, the $c$-bundle is mereologically constant: if the c-bundle exists at some moment $t$, each of its proper parts must also exist at $t{ }^{15}$ Thus, if the $c$-bundle exists at $t_{1}$, the nuclear tropes of substance $i$ and the $n$-bundle of $i$ must also exist at $t_{1}$. Hence, the temporal location of the $c$-bundle is a proper or improper sub-location of the temporal location of the $n$ bundle. The $c$-bundle cannot exist, when the $n$-bundle does not exist. When we add into this that the location of contingent trope $u$ is determined by the location of the $c$-bundle, it follows

13 As we will see just below, this does not yet entail that the nuclear tropes and substance $i$ must have the same (spatial) size. However, it will be cleared up that it is not difficult to come to this conclusion.

14 SNT assumes that $n$-bundles are as trope aggregates identical with individuals figuring in the basic spatio-temporal relations. Similarly, c-bundles are identical with individuals. Hence, in addition to mereologically inconstant individuals (substances), tropes form mereologically constant individuals (identified with trope aggregates), i.e., c-bundles and n-bundles. For the sake of brevity, we simply speak about $c$-bundles and $n$-bundles. 15 Trope aggregates satisfy the thesis of Strong Mereological Essentialism (SME) formulated by Simons $(1987,272)$ in modal temporal mereology. 
that trope $u$ can exist only when the $n$-bundle exists. This is an important result for the persistence of $i$.

Since the $c$-bundle is a trope aggregate having the nuclear tropes of $i$ as its constituents, the spatial location of the c-bundle must include the spatial location of the nuclear tropes. Hence, at each moment in which the c-bundle exists, the spatial region occupied by the $n$ bundle is a proper or improper part of the spatial region occupied by the $c$-bundle. ${ }^{16} \mathrm{In}$ other words, the $c$-bundle cannot be spatially smaller than the $n$-bundle.

However, it does not follow from this that the c-bundle cannot be even enormously larger in space than the $n$-bundle and that trope $u$ cannot be (in part) widely distant from the $n$ bundle and the rest of substance $i$. For that, we must argue further that the c-bundle and $n$ bundle have exactly the same spatial location at each moment of their existence. The argument to this conclusion is comparatively straightforward but requires that each simple substance is assumed to be a powerful particular. Powerful particulars are particular substances whose monadic features are all dispositional (like mass, spin, and electric charge) and correspond to the specific causal powers of a powerful particular (e.g., to attract gravitationally other objects with a mass). According to the best a posteriori information we have on reality, all fundamental substances are powerful particulars. ${ }^{17}$ As simple substances, powerful particulars have a centre of influence of their causal powers and only an approximate size determined by their total causal powers. ${ }^{18}$ The shape and size of a macro-object is determined by the arrangement of the different kinds of powerful particulars constituting the macro-object.

The constituent tropes of a simple substance $i$ determine its causal powers and have exactly the same centre of influence as these causal powers. Individual tropes do not have a set of causal powers, but rather contribute to determining the causal powers of a powerful particular. Hence, tropes have a centre of influence but fail to have any definite size. Thus, the

16 Concrete entities (e.g., tropes) and their aggregates satisfy the principle of Expansivity formulated by Parsons $(2007,213)$ with respect to their spatial location: the spatial location of a whole must include the spatial locations of its proper or improper parts.

17 For example, Stephen Mumford (2006) has defended this claim.

18 Harré $(1970$, ch. 11) calls simple substances 'point centres of influence'. A point centre of influence has only dispositional features and an approximate size, which is determined by its dispositional features and the net attractive and repulsive forces between the point centre and other simple substances (i.e., point centres). In order to distinguish between simple substances and their spatial locations, we prefer to call the latter 'centres of influence' of simple substances. We do not assume that simple substances have point-like spatial locations. 
spatial location of trope $u$ is the same as its centre of influence. Although not necessarily pointlike, the centres of influence do not have different sizes. Thus, the location of the nuclear tropes of $i$ is the same as the centre of influence of the $n$-bundle and the location of contingent trope $u$ of $i$ is the same as the centre of influence of the $c$-bundle. The latter centre of influence must include the former at each moment of the existence of $u$. Since the centres of influence do not have different sizes, contingent trope $u$ of $i$ and the nuclear tropes of substance $i$ must be spatially co-located at each of moment in which they all exist.

Thus, with the help of the further assumptions we consider plausible, we can say that the spatial region occupied by each contingent trope $u$ and $c$-bundle of a simple substance $i$ is exactly the same as the spatial region occupied by the $n$-bundle of $i$ when trope $u$ exists. Previously it was concluded that the temporal region occupied by each contingent trope $u$ is a proper or improper part of the temporal region occupied by the $n$-bundle. The locations of the different $c$-bundles (and contingent tropes) of $i$ are independent of each other but co-ordinated by the location of nuclear tropes: each contingent trope can exist some time interval shorter than the nuclear tropes of a substance but when a contingent trope exists it is necessarily and exactly co-located with the nuclear tropes (and the whole substance). The contingent tropes of $i$ do not exist when and where $i$ does not exist.

It is important to realise that according to the SNT there is a difference in the mereological nature of simple substances, $n$-bundles and $c$-bundles. Every $n$-bundle and $c$ bundle is mereologically constant as an aggregate: they cannot change their trope parts without destruction. By contrast, simple substances are mereologically inconstant. These explain the possibility of alteration (Kurtz's thesis 1) and persistence respectively. Since there can be change in the contingent tropes and c-bundles of a simple substance, the simple substance may change its parts and still remain identical over time. The possibility of change is explained by both the inconstancy of the simple substance and the constancy of the $n$-bundle. As a simple substance can change its trope parts, it can instantiate incompatible property tropes at different moments of time. However, it is the same substance, it survives change because it persists due to the mereologically constant $n$-bundle. So there is an entity, the substance that is the proper subject of change.

Recall that Kurtz's non-negotiable thesis 4 states that objects cannot have incompatible properties. According to the SNT, this should be specified: simple substances can have properties over time that would exlude each other at the same time. In the next section, we will 
show that this is possible without time-relativization, i.e., without denying Kurtz's thesis 3.2 in our modified form (3.1).

\section{No Temporal Relativization of Truthmakers}

According to the SNT, the contingent intrinsic features of a simple substance $i$ are determined by its contingent tropes. SNT gives a special kind of 3D'ist answer to the problem of temporary intrinsics. The facts about time are by no means irrelevant to the truth of the proposition that a simple substance possesses a contingent intrinsic feature $F$ : each such proposition is temporally relativized in standard cases. By contrast, neither the truthmaker of the proposition (contingent trope $u$ possessed by the substance) nor the relation between the trope and the substance is temporally relativized.

First, no bundle where a constituent trope of $i$ occurs is internally temporally modified. As a concrete particular, each trope constituent of a substance has a specific spatio-temporal location. While the $n$-bundle grounds the location of nuclear tropes, the location of a contingent trope $u$ is grounded by the location of its $c$-bundle. The spatio-temporal location of the $n$-bundle and $c$-bundle is determined by relational tropes connecting them either with other trope aggregates or space-time points. ${ }^{19}$ Hence, spatio-temporal location is extrinsic to these trope bundles and their constituent tropes.

Secondly, this does not entail that the relation between a trope and its substance (ontic predication) is eventually temporally relativized in the SNT. Let us take up nuclear tropes first. According to the SNT, a nuclear trope $u$ is a property of substance $i$ iff [1] $u$ is strongly rigidly dependent on $i$, [2] necessarily, if $u$ exists, $u$ is a proper part of $i$, and [3] $u$ is necessarily compresent with $i$. As was shown in the previous section, all these conditions hold for every nuclear trope of a simple substance for the following reasons. [1] If $u$ is a nuclear trope, its existence entails the existence of $i$ because it is strongly rigidly dependent on the other nuclear tropes of $i$ and thus on $i$ itself. [2] This also involves that $u$ is a proper part of $i$. The formal relation of rigid dependence is atemporal. In addition, the proper-parthood relation between

19 Depending on the favoured theory of space-time, the trope bundles are either the sole relata of spatiotemporal relations (Space-time Relationalism) or together with space-time points (Space-time Substantivalism). See Sklar (1974) for further discussion of these alternative ontologies of space-time. 
nuclear trope $u$ and the substance holds atemporally, i.e., without temporal relativization. So [1] and [2] do not involve temporal relativization. As nuclear trope $u$ is a part of the $n$-bundle of $i$, the location of $u$ is grounded by the location of the $n$-bundle. Trope $u$, the $n$-bundle and $i$ must also have the same centre of influence and they must exist when any of them exists. So $u$ fulfils condition [3].

Assume now that $u$ is a contingent trope of substance $i$. Trope $u$ is a property of substance $i$ iff [1] $u$ is strongly rigidly dependent on $i$, [2] necessarily, if $u$ exists, $u$ is a proper part of $i$, and [3'] necessarily, the temporal region that $u$ occupies is a proper or improper part of the temporal region occupied by $i$, whereas the spatial location of $u$ coincides with the location of $i$ at each moment of $u$ 's existence. Conditions [1] and [2] hold for every contingent trope of $i$ for the same reasons as in the case of its nuclear tropes (with the irrelevant difference that a contingent trope is not SRD on the other nuclear tropes but on every nuclear trope of $i$ ). So no contingent trope is temporally relativized either. As we argued above, condition [3'] holds for $u$ mainly because the $c$-bundle having $u$ as its constituent satisfies [3']. The temporal condition for $u$ in [3'] is explained by three factors. (1) As trope aggregates, the c-bundle and the $n$-bundle are mereologically constant and Strong Mereological Essentialism holds for them. This in turn entails that the $c$-bundle can exist only when the $n$-bundle exists. (2) These trope aggregates are fundamental entities figuring in spatio-temporal relations. (3) Thus, the temporal location of $u$ relative to the $n$-bundle and $i$ is determined by the temporal location of the $c$ bundle relative to the $n$-bundle. Hence, trope $u$ can exist only when the $n$-bundle and substance $i$ exist. So its temporal location is the proper or improper sub-location of the temporal location of the $n$-bundle, and thus of substance $i$. Temporal condition in [3'] is fulfilled.

However, somebody might still insist that [3'] involves the temporal relativization of trope $u$ (a temporary intrinsic) and the $c$-bundle. They are related to substance $i$ and the $n$ bundle by temporal sub-location. So trope $u$ and the $c$-bundle are temporary parts of $i$ if one wants to talk about temporary parts.

Our reply to this worry is that the sub-location relation and temporary parthood do not mean that the ontic predication of trope $u$ or a temporary intrinsic is temporally relativized. The point is simple. First, temporal sub-location is not time-relativization; rather, it is atemporal part-whole relation, sharing temporal location. ${ }^{20}$ Secondly, temporary parthood is theoretically

20 Note that also worm-theory has to admit that a substance as a mereological sum and its temporary intrinsics share temporal sub-location without commitment to temporal relativization. 
redundant in the SNT: it does not do any explanatory work but is accounted for by [1], [2], and [3'] instead. ${ }^{21}$

In the case of simple substances, the SNT solves the problem of persistence by accepting Kurtz's theses 1 (alteration), 2 (survival), 5 and 6, and specifying 3 (atemporary intrinsics) together with 4 (objects cannot have incompatible properties). The specified thesis 3 is our proposition 3.1: having properties is not relativized to time. First, according to the SNT, simple substance $i$ can instantiate, at the distinct moments of its existence, contingent property tropes that would exlude each other at the same time (specified 4 and thesis 5). Simple substances are mereologically inconstant. Second, numerically identical substance $i$ survives change because its nuclear tropes persist. The $n$-bundle of every simple substance is mereologically constant as an aggregate. So there is something that remains identical in alteration: the simple substance (theses 2 and 6). Third, as we just showed, neither property tropes nor the possession of tropes are temporally relativized. As a contingent trope $u$ (and the corresponding $c$-bundle) can have more restricted temporal location than substance $i, u$ need not be instantiated by $i$ at each time of its existence (thesis 3.1). Facts about time are relevant to the truth of the proposition that a substance $i$ possesses intrinsic feature $\mathrm{F}$ determined by trope $u$ (contra 3.2).

SNT gives also a non-relational analysis of ontic predication, i.e., no new relational entities (e.g., relational tropes) are introduced to account for ontic predication. We can explain ontic predication by means of formal relations and the entities that already explain the location of tropes. Hence, both in the case of nuclear and contingent tropes, we obtain a non-relational analysis of ontic predication in terms of formal relations (parthood, rigid existential dependence) and the grounded internal relations of compresence and sub-location. ${ }^{22}$

This leads us to the discussion of Bradley's regress.

21 Each contingent trope $u$ is a part of substance $i$ at some time $t: \mathrm{u}<_{\mathrm{t}} \mathrm{i}$. As a consequence, both $u$ and substance $i$ exist at $t: \mathrm{E} !_{\mathrm{t}} \mathrm{u} \wedge \mathrm{E} !_{\mathrm{t}} \mathrm{i}$, cf. Simons $(1987,179)$. Trope $u$ is a proper part of $i$ at each moment of its existence: if and only if trope $u$ exists at some time t', $u$ is a proper part of $i$ at $\mathrm{t}^{\prime}$. However, in the SNT, the existence of a trope at $\mathrm{t}^{\prime}$ is explained in terms of temporal location. Thus, $u$ is a temporary part of $i$ because the temporal location of the corresponding $c$-bundle is a part of the temporal location of $i$.

22 Grounded internal relations are relational features of entities determined by the properties and/or relations instantiated by the same or distinct entities (e.g., the proper parts of the first entities). Cf. Author () for a more comprehensive account of the different kinds of grounded internal relations. 


\section{No Bradley's Regress}

It was perhaps F.H. Bradley (1846-1924) who first presented the problem that bears the title of his regress in Appearance and Reality (1893). It seems that Bradley did not distinguish between internal and external relations but thought that the regress gets started if we introduce distinct qualities that form a plurality, and therefore, must stand in some relation to each other. ${ }^{23}$ By contrast, it is nowadays customary to delimit the regress problem to (the ungrounded) external relations. In what follows, we first present the contemporary formulation of the problem and then give a principled answer to it.

The contemporary problem of Bradley's regress can be systematically described as follows. Let us first suppose that external relations are further entities to their relata. Let us make another assumption that entities $a$ and $b$ are related by external relation $C$. As $C$ is an external relation, the existence of $a$ and $b$ does not entail that $a$ and $b$ are connected by $C$, i.e., that $C a b$. Similarly, it seems that the existence of relation $C$ does not entail that $C a b$, i.e., that $a$ and $b$ are connected by $C$. Therefore, we must postulate another external relation $C$ in order to explain why $a$ and $b$ are connected by $C$. However, the existence of relation $C^{\prime}$ does not entail that its relata $a, b$ and $C$ are connected by $C^{\prime}$. As a consequence, we must introduce a still further external relation and an infinite regress gets started. The regress seems to be vicious because we must postulate an infinite number of relations in order to explain why two entities are connected by a single relation.

Bradley's regress is a general problem concerning relations. But for this very reason, it threatens every account of ontic predication and temporary intrinsics that employs relations, temporally relativized intrinsic properties, for example. These accounts are not good explanations if they collapse into vicious infinite regress.

A reply to Bradley's regress in general is to deny that external relations are connected to their relata by a further relation. Relations are considered to be entities that connect entities without the help of any further entity. Two fundamentally different kinds of such answers have been presented in the literature. First, factualists have claimed that holding of a relation is a

23 What Bradley's original problem exactly is is a difficult interpretative question. There is no room in this paper to address that problem. Therefore it is not reasonable to consider Bradley's original regress here. For a historical discussion of it, see Candlish and Basile (2009, ch. 6). 
further entity in its own right, i.e., a relational fact (cf. Armstrong 1997). Secondly, another answer is to maintain, denying one premise of the above argument, that the existence of an external relation already entails that the entities occurring in the relation are connected by the relation at issue (Simons 2003; Maurin 2009). We will defend the latter kind of answer and argue that our version of it avoids Bradley's regress.

Our answer to Bradley's regress in the specific case of temporary intrinsics is simple. First, trope aggregates are bundled by strong rigid and generic dependences. These dependences as well as parthood are formal ontological relations, which are not further entities to their relata. As such that aspect of the first assumption of Bradley's regress that relations are further entities to their relata is denied. Secondly, external relations are connected to their relata by formal relations. The regress halts at formal relations, which are not distinct entities from their relata.

Regarding the first answer, it is assumed in the SNT that all formal relations are ungrounded internal relations: if formal relation $R$ connects $a$ and $b$, the existence of relata $a$ and $b$ entails that $a$ and $b$ stand in formal relation $R$. Hence, Bradley's regress does not directly apply to them because it is assumed in it that the existence of relata does not entail that they are connected with the relation. Besides, formal relations hold on the basis of the existence of their relata: different kinds of formal relations constitute a network and given that entity $e$ exists, its position in the network of formal relations of every kind must already be fixed. For the present purposes, suffices it to take up two types of formal relation. On the one hand, mereological relations specify how a given entity $e$ exists as a constituent of the world (e.g., as a part of another entity). On the other hand, different kinds of relations of existential dependence specify how a given entity $e$ can exist as a constituent of the world together with the other entities. It must be a built-in feature of any entity $e$ that its mereological relations and existential dependencies to all other entities are fixed. Consequently, formal relations are not further relational entities but just relational predications made true by their relata. Formal relations hold on the basis of the existence of their relata. Hence, they cannot generate Bradley's regress. The second point is that the spatio-temporal relations between trope bundles are relational tropes. ${ }^{24}$ These relations are thus extra entities to their relata. However, the relational tropes are

24 The relata of relational tropes must not be strongly rigidly dependent on each other. This is necessary because otherwise a contingent trope SRD on two nuclear tropes, which are SRD on each other, would be a relational trope, cf. Author A(). 
related to their relata by strong rigid dependence. Given that relational trope $R$ exists, $R$ is both rigidly dependent on its relat $a$ and $b$ and $a$ and $b$ are connected by $R$. Thus, Bradley's regress stops at these strong rigid dependencies because they are formal relations and it does not threaten the analysis of ontic predication in the SNT. ${ }^{25}$ Hence, we avoid the contemporary Bradley's regress problem because the relations introduced in the SNT are relational tropes that connect their terms due to their own existence.

\section{Coincidence, Fission, Fusion, and Undetached Parts}

The problem of coincidence is that there are cases where two numerically distinct concrete entities share the same spatial location, which violates the principle that there cannot be spatially co-located distinct concrete objects. Normally these cases discussed in the literature, for example 'the Statue and the Lump of Clay', concern (highly) complex entities (see Sider 2001, 5-6, 142). As such, they do not create any problem for the SNT, which is restricted to simple substances. However, a principled answer can be given that the SNT permits coincidence of concrete entities. According to it, tropes, their bundles ( $c$ and $n$ ) and simple substances are spatially co-located with each other.

The question about fission and fusion, which has been much discussed especially in the literature on personal identity (specific case of persistence) ${ }^{26}$, does not really rise in the SNT. According to it, simple substances cannot divide or fuse and it is not even possible that contingent tropes move to another simple substance. Every trope of a simple substance is strongly rigidly dependent on the nuclear tropes of the substance. So they cannot occur in other substances, which all these possibilities would require. Besides, fission and fusion cases are about (highly) complex substances and are as such out of the scope of this paper.

The same point concerns Wiggins' puzzle of Tibbles and Tib. Tibbles is a cat and Tib its proper part without the tail. When Tibbles loses her tail, the question is: do two previously distinct entities coincide after the tail is lost? This puzzle is thus a specific case of what Sider

25 For a similar conception of relational tropes, cf. Simons (2003); Author A. () and Maurin (2009).

26 Here we allude to Parfit's problem of dividing personality and the traditional problem of the Ship of Theseus, for example. For a brief exposition of these problems, see Sider (2001, 143-147). In the fission and fusion cases, entities divide or fuse and the question is how to determinate identity relations in these scenarios. 
(2001, 142) calls 'Undetached Parts'. In the SNT, something similar can happen because a simple substance may loose its proper parts, i.e., contingent tropes; consider the case that first we have a contingent trope and a $c$-bundle with a $n$-bundle as the proper part of the $c$-bundle and then the contingent trope goes out of existence. However, according to the SNT identity relations are clear in this case. By Strong Mereological Essentialism, the c-bundle is also destroyed when the contingent trope ceases to be. But the $n$-bundle (and the substance) remains intact. So there is no coincidence after the $c$-bundle goes out of existence.

\section{Conclusion}

In this paper, we have defended a novel 3D for the standard objections to the 3D'ist accounts for the persistence of simple substances. According to the Strong Nuclear Theory, simple substances are bundles of tropes, which are particular dispositional properties. A simple substance must have one or more tropes that are necessary to it. If there are two or more of these necessary tropes, they are strongly rigidly dependent on each other and form the $n$-bundle

of the substance. As an object of kind K, the substance must have tropes of the certain distinct determinable kinds $D_{1}, \ldots D_{k}$. If its nuclear tropes do not satisfy this condition, the substance has to have at least one contingent trope (and correspondent $c$-bundle). These contingent tropes are strongly rigidly dependent on the nuclear tropes of the substance, but not vice versa.

SNT accounts for the cross-temporal identity of a simple substance by the persistence of its nuclear tropes or $n$-bundle. As such, it is a 3D'ist position in Della Rocca's definition of the distinction between 3D and 4D: it explains persistence in virtue of the same entities. SNT is also capable of accounting for the persistence of simple substances in the narrow sense. It is possible that a simple substance changes qualitatively because there can be alteration in its contingent tropes even if it has to have contingent tropes of certain kinds. However, the SNT does not suffer from two problems often attributed to 3D: temporal relativization of ontic predication or temporary intrinsics and Bradleys' regress.

SNT does not relativize ontic predication to time. According to it, a simple substance must have all of its nuclear tropes as its proper parts in every moment it exists. One of the neat features of the SNT is that trope aggregates, not individual tropes are minimal entities instantiating basic spatio-temporal relations. Tropes figure in these relations only derivatively. 
This has significant consequences for the analysis of temporary intrinsics. In the SNT, the temporal location of a simple substance in relation to each of its (possible) contingent tropes is analysed in terms of the temporal location of the $c$-bundle having the contingent trope as its constituent in relation to the $n$-bundle of the substance. Thus, no contingent trope is directly relativized to time in the SNT. Besides, there is no danger of indirect temporal relativization either because the c-bundle and the $n$-bundle co-ordinate the temporal location of the contingent trope in relation to the substance. The $c$-bundle is mereologically constant. Thus, whenever it exists, all its proper parts must also exist. Since the $n$-bundle is one of those proper parts, the $n$-bundle has to exist when the c-bundle exists. In other words, they are properly or improperly sub-located in time. This also means that the temporal region occupied by the contingent trope is a proper or improper sub-location of the temporal region occupied by the $n$ bundle and the substance. The sub-location relation does not involve time relativization because it amounts to sharing temporal region.

SNT does not collapse into Bradley's regress either. Bradley's regress presupposes that relations are external and additional entities to their relata. First, the SNT construes simple substances by formal ontological relations (existential dependences and parthood) and relational tropes explain the spatio-temporal relations between the parts of substances. Formal relations are not further entities to their relata and Bradley's regress does not get off the ground. Secondly, in the SNT, relational tropes are distinct entities from their relata. So Bradley's regress threatens it in this respect. However, the regress is not generated in the end because relational tropes are related to their relata by formal ontological relations. Bradley's regress stops at them as they are not further entities to their relata.

\section{Acknowledgements}




\section{References}

Armstrong, D. M. (1997) A World of States of Affairs. Cambridge University Press, Cambridge.

Bradley, F. H. (1893). Appearance and Reality. Oxford University Press, Oxford.

Campbell, K.K. (1990). Abstract Particulars. Basil Blackwell, Oxford.

Candlish, S. \& Basile, P. (2009). Francis Herbert Bradley. In: Zalta, E.N. (Ed.) The Stanford

Encyclopedia of Philosophy. Spring 2009 Edition. Available from http://plato.stanford.edu/archives/spr2009/entries/bradley/.

Crisp, T.M. \& Smith, D.P. (2005). 'Wholly present' defined. Philosophy and the Phenomenological Research 71(2): 318-344.

Denkel, A. (1996). Object and Property. Cambridge University Press, Cambridge.

Denkel, A. (1997). On the Compresence of Tropes. Philosophy and Phenomenological Research LVII(3): 599-606.

Harré, R. (1970). The Principles of Scientific Thinking. Macmillan, London.

Haslanger, S. (1989). Endurance and Tempory Intrinsics. Analysis 49: 119-125.

Haslanger, S. \& Kurtz R.M. (Eds.) (2006). Persistence - Contemporary Readings. MIT press, Cambridge Ma.

Hawley, K. (2001). How Do Things Persist. Oxford University Press, Oxford.

Hawthorne, J. (2008). Three-Dimensionalism vs. Four-Dimensionalism. In: Sider, T., Hawthorne, J. \& Zimmerman, D. (Eds.) Contemporary Debates in Metaphysics (pp. 263-282). Oxford University Press, Oxford.

Kurtz, R.M. (2006). Introduction to Persistence: What's the Problem? In: Haslanger, S. \& Kurtz, R.M. (Eds.) Persistence - Contemporary Readings (pp. 1-26). MIT Press, Cambridge Ma.

Lewis, D.K. (1986). On The Plurality of the Worlds. Basil Blackwell, Oxford.

Lowe, E.J. (1998). The Possibility of Metaphysics - Substance, Identity and Time. Clarendon Press, Oxford.

Maurin, A-S. (2002). If Tropes. Kluwer Academic Publishers, Dordrecht.

Maurin, A-S. (2009). Trope Theory and the Bradley's Regress. Synthese (in press).

Mellor, D.H. (1981). Real Time. Cambridge University Press, Cambridge.

Mumford, S. (2006). The Ungrounded Argument. Synthese 149: 471-489. 
Parsons, J. (2007). Theories of Location, In: Zimmerman, D. (Ed.) Oxford Studies in Metaphysics vol. 3 (pp. 201-232). Oxford University Press, Oxford.

Sider, T. (2001). Four Dimensionalism - An Ontology of Persistence and Time. Oxford University Press, Oxford.

Sider, T., Hawthorne, J. \& Zimmerman, D. (Eds.) (2008). Contemporary Debates in Metaphysics. Blackwell Publishing, Oxford.

Simons, P.M. (1987). Parts - a Study in Ontology. Clarendon Press, Oxford.

Simons, P.M. (1994). Particulars in Particular Clothing - Three Trope Theories of Substance. Philosophy and Phenomenological Research LIV(3): 553-575.

Simons, P.M. (2003). Tropes, Relational. Conceptus 35: 53-73.

Simons, P.M. (2004). Extended Simples: A Third Way Between Atoms and Gunk. Monist 87: 371-384.

Sklar, L. (1974). Space, Time, and Spacetime. University of California Press, London. Van Inwagen, P. (1990). Four-dimensional Objects. Nous 24: 245-255.

Williams, D. C. (1953a). On the Elements of Being I. Review of Metaphysics 7: 3-18.

Williams, D. C. (1953b). On the Elements of Being II. Review of Metaphysics 7: 171-192.

Zimmerman, D. (Ed.) (2007). Oxford Studies in Metaphysics vol.3. Oxford University Press, Oxford. 